\title{
Influence of Handover Mechanisms and Transmission Delay Difference on SFN Gain
}

\author{
David Plets, Wout Joseph, \\ Leen Verloock, Luc Martens \\ Department of Information Technology \\ Ghent University/IBBT \\ Ghent, Belgium \\ Email: david.plets@intec.ugent.be
}

\author{
Pablo Angueira \\ University of the Basque \\ Country (UPV/EHU) \\ Bilbao, Spain
}

\author{
José Antonio Arenas \\ Telefónica Móviles España \\ Spain
}

\begin{abstract}
Single Frequency Networks (SFNs) are often deployed for their optimal frequency reuse and the more homogeneous distribution of the field strength in the covered area. Different methodologies have already been proposed to calculate the so-called SFN gain over Multi Frequency Networks (MFNs), but so far, the influence of (MFN) handover mechanisms on the gain values has not yet been investigated. Also, it can be expected that in SFNs, the gain values will depend on the transmission delay difference of the signals from the different transmitters in the SFN. This paper will first assess the influence of a handover mechanism on previously obtained SFN gain values. Secondly, it will be investigated if the transmission delay difference is a good predictor for the SFN gain. This paper further clarifies the SFN concept from a network planner's point of view and aids in understanding what a network planner should take into account when deploying an SFN.
\end{abstract}

\section{INTRODUCTION}

Operators always try to keep the total cost of a network deployment as low as possible. Theoretically, a Single Frequency Network (SFN) delivers the same quality as a Multi Frequency Network (MFN), but with a reduction in transmitting power. This leads to a lower number of required base stations and thus a lower cost. Available literature mostly deals with SFN gain in an optimistic way, although the quality of the reception may be impaired by self-interference and synchronization and equalization problems. In [1], the authors have proposed a methodology to calculate a meaningful value of the SFN gain and applied it to a real DVB-H (Digital Video Broadcasting - Handheld) network. In this paper, extensions to the used approach will be investigated. Firstly, SFN gains will be determined based on a comparison of the SFN scenario with a realistic MFN scenario that takes into account handover mechanisms, an approach that to our knowledge has not yet been investigated in literature. Secondly, the influence on the SFN gain of the transmission delay difference observed at the receiver will be investigated.

\section{TRANSMISSION CONFIGURATION}

The DVB-H SFN consists of 3 transmitters (Tx) in the city of Ghent, Belgium, a mostly suburban environment. The SFN consists of three base station antennas (BS) and operates at a frequency of $602 \mathrm{MHz}$. The channel bandwidth is $8 \mathrm{MHz}$. Fig. 1 shows the map of Ghent with the location of the

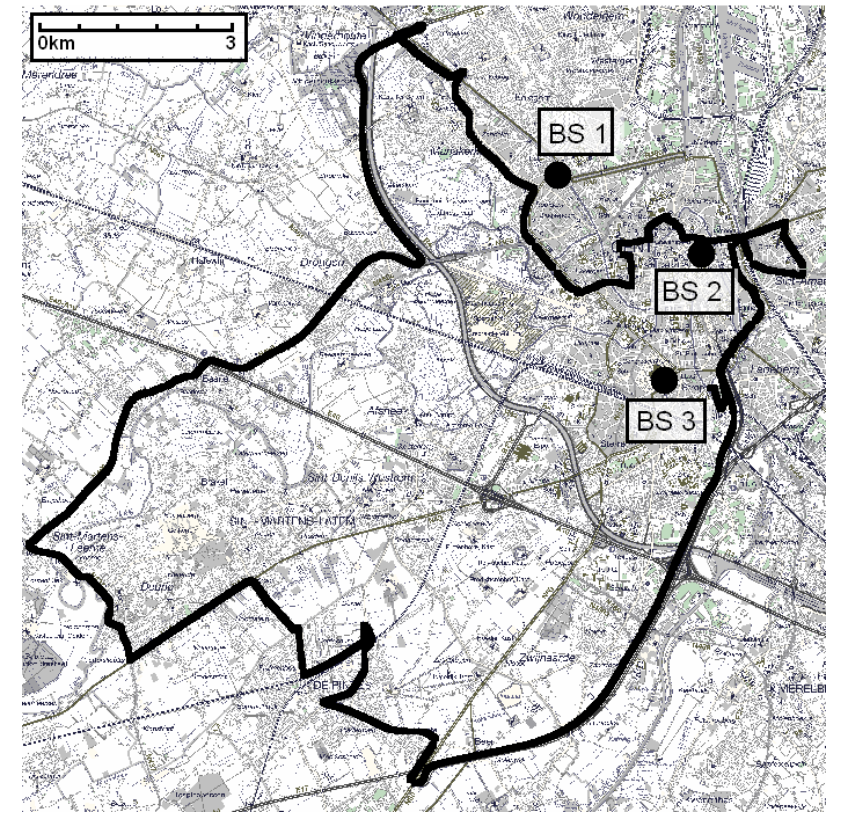

Fig. 1. Map of Ghent with the three base stations (black dots) and indication of the measurement route.

three base stations marked with black dots. All Txs are omnidirectional and vertically polarized. The heights of these Tx are $\mathrm{hTx}=57 \mathrm{~m}, \mathrm{hTx}=64 \mathrm{~m}$, and $\mathrm{hTx}=63 \mathrm{~m}$, respectively. The EIRP (Equivalent Radiated Power) used for these Tx is $36.62 \mathrm{dBW}, 39.93 \mathrm{dBW}$, and $40.90 \mathrm{dBW}$, respectively. 16QAM $1 / 2,4 \mathrm{~K}$, MPE-FEC $7 / 8$ is used with a guard interval of $1 / 8$, corresponding with a useful bit rate of $9.68 \mathrm{Mbps}$. The characteristics of the transmitters are summarized in Table I.

\section{Measurement Equipment and Available MEASUREMENTS}

To determine the SFN gain SFNG, Modulation Error Ratio (MER) measurements are performed with a commercial tool consisting of a PCMCIA (Personal Computer Memory Card International Association) card with a small receiver antenna $\mathrm{Rx}$. The PCMCIA card is plugged into a laptop, which is used to perform and process the measurements. Every $0.5 \mathrm{~s}$, a sam- 


\begin{tabular}{|r|r|r|}
\hline Constellation & \multicolumn{2}{|c|}{ 16-QAM 1/2 } \\
\hline MPE-FEC rate & \multicolumn{2}{|c|}{$7 / 8$} \\
\hline Guard interval & \multicolumn{2}{|c|}{$1 / 8$} \\
\hline FFT mode & \multicolumn{2}{|c|}{$4 \mathrm{~K}$} \\
\hline Useful bit rate [Mbps] & \multicolumn{2}{|c|}{9.68} \\
\hline \hline & Height [m] & EIRP [dBW] \\
\hline BS 1 & 57 & 36.62 \\
\hline BS 2 & 64 & 39.93 \\
\hline BS 3 & 63 & 40.9 \\
\hline
\end{tabular}

TABLE I

CHARACTERISTICS OF DVB-H TRANSMITTING NETWORK.

ple is recorded. The tool logs parameters as MER (modulation error ratio), FER (Frame Error Rate), MFER (Multi-Protocol Encapsulation FER), and electric-field strength. MFER is the ratio of the number of residual erroneous frames (i.e., not recoverable) and the number of received frames [2]. FER is the ratio of the number of erroneous frames before MPE-FEC correction and the number of received frames [2]. Location and speed are recorded with a GPS device. Measurements are performed inside a small van at a height of about $1.5 \mathrm{~m}$ above ground level.

A route of about $50 \mathrm{~km}$ is driven to evaluate the SFN gain. The route stretches from the very centre of Ghent to the municipalities that surround Ghent (see Fig. 1). The analysis in this paper is performed for mobile reception at velocities of $50-70 \mathrm{~km} / \mathrm{h}$ (common in Belgium).

Four scenarios are investigated: all three transmitters active (and synchronized) as an SFN at the same time ( Scen $_{\mathrm{SFN}}$ ), and each of the individual transmitters active, while the other two are inactive $\left(\mathrm{Scen}_{1}, \mathrm{Scen}_{2}\right.$, and $\left.\mathrm{Scen}_{3}\right)$. About 10,000 samples are collected for each scenario.

\section{SFN GAin Definition AND NOVEl Approaches to THE SFN GAIN CALCULATION}

The measured route is divided into segments of $100 \mathrm{~m}$. After spatially aligning the segments for the four scenarios, the MER values in a certain segment are compared for the different scenarios. In [1], the SFNG in a segment is defined as the MER in that segment in SFN mode (all three transmitters active) minus the maximum MER in that segment in MFN mode (only one transmitter active).

$$
\mathrm{SFNG}=\mathrm{MER}_{\mathrm{Scen}_{\mathrm{SFN}}}-\max \left(\mathrm{MER}_{\mathrm{Scen}_{\mathrm{i}}}\right),[\mathrm{dB}]
$$

with $\mathrm{i}=1,2$, or 3 (only one transmitter active).

However, this assumes an ideal MFN scenario where the receiver always automatically switches to the transmitter providing it with the highest MER (best serving transmitter) within the $100 \mathrm{~m}$-window. In reality, handover mechanisms will be used to avoid the energy-consuming ping-pong effect [3] at the receiver. Although the gain values proposed in [1] are very valuable, they might thus be too pessimistic (for SFNs), since the MER values observed in the MFN scenario might be overestimated. Therefore, this paper will determine gain values based on the use of a handover mechanism. This handover mechanism works as follows [3]. The receiver is assumed to connect to the transmitter, which has provided it with the highest Modulation Error Ratio (MER) within the last 500 $\mathrm{m}$-window. However, handovers are not executed when the currently serving transmitter already provides a MER value higher than the MFER1\% value, because then, switching to another transmitter is not necessary to maintain a perfect reception quality. The moving $500 \mathrm{~m}$-window is evaluated each $100 \mathrm{~m}$, which means that the subsequent windows overlap for $80 \%$. We have to use distance-based windows instead of time-based windows, since the four drives (of Fig. 1) obviously have a different time-location relation. Assuming an average speed between 50 and $70 \mathrm{~km} / \mathrm{h}$, the $500 \mathrm{~m}$-window corresponds to approximately $25-35 \mathrm{~s}$, with possible handovers each 5-7 s. The SFNG in a segment now becomes:

$$
\mathrm{SFNG}=\mathrm{MER}_{\mathrm{Scen}_{\mathrm{SFN}}}-\left(\mathrm{MER}_{\mathrm{Scen}_{\mathrm{i}}}^{\mathrm{HO}}\right),[\mathrm{dB}]
$$

where the superscript 'HO' indicates that the MER value is obtained from Scenario i, as determined by the handover algorithm.

Also in [1], the changes in quality of service as a function of the intensity of the SFN overlapping $E_{\text {diff }}$ are provided. $E_{\text {diff }}$ is the difference between the electric-field strength due to the dominant transmitter (i.e., the transmitter causing the highest MER) and the electric-field strength due to the second strongest transmitter:

$$
\mathrm{E}_{\mathrm{diff}}=\mathrm{E}_{\text {median }}^{\text {DominantBS }}-\mathrm{E}_{\text {median }}^{\text {SecondStrongestBS }}[\mathrm{dB}]
$$

However, it can be expected that there is also a significant correlation between the transmission delay difference observed at the receiver and the SFN gain. Although DVB-H introduced a guard interval to cope with these delays, performance degradation has already been observed for signals arriving within the guard band. It will be investigated if this measure is a better predictor for SFN gain than the previously used overlapping degree of the transmitters.

\section{Results}

In this section, the influence of taking into account a handover mechanism for SFN gain calculations will first be discussed. Then, the link between the SFN gain (without handover mechanism) and the transmission delay difference experienced at the receiver will be analyzed.

\section{A. Influence of Handover Mechanism on SFNG}

Fig. 2 shows the SFN gain along the measurement trajectory according to the method proposed in [1] ('always best serving transmitter' or 'ideal scenario', red), and according to the method that implements the proposed handover mechanism ('realistic handover scenario', see Section IV, black). When using the handover mechanism, the median gain along the entire trajectory increases from $0.76 \mathrm{~dB}$ to $1.13 \mathrm{~dB}$. This increase 
is limited due to the limited number of handovers executed, and also due to the fact that even in [1], a segmentation has been applied (segment length of $100 \mathrm{~m}$ ) for small-scale fading cancelling and synchronization purposes. This segmentation already incorporates a handover mechanism, because within one segment (100 m or 5-7 s), handovers are impossible.

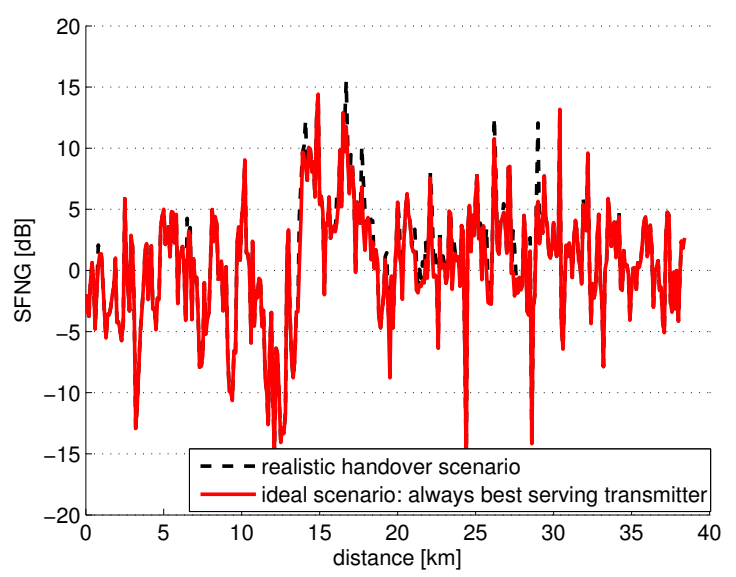

Fig. 2. SFNG $[\mathrm{dB}]$ along the trajectory for a realistic handover scenario and for a scenario where the receiver always chooses the best serving transmitter.

Table II shows the median SFNG SFNG med $_{\text {in the handover }}$ scenario and in the ideal scenario for the four different reception scenarios [1] and four different overlapping categories [1]. For better reception qualities ('good' and 'perfect'), no increase in median SFNG is noticed, because in this case, no handovers are executed, due to one transmitter being dominant over the other two. This corresponds with the case of low overlap between the transmitters: $6<\mathrm{E}_{\text {diff }}$.

When the dominant transmitter provides the receiver with only a doubtful or low quality signal, handovers are more likely: the receiver receives comparable signals from the two most dominant transmitters $\left(\mathrm{E}_{\mathrm{diff}}<6\right)$. When the number of handovers is then being limited by the handover algorithm, the median SFNG increases: from $2.20 \mathrm{~dB}$ to $2.42 \mathrm{~dB}$ for doubtful reception and from $2.24 \mathrm{~dB}$ to $3.08 \mathrm{~dB}$ for low quality reception (see Table II). Expressed in terms of $\mathrm{E}_{\text {diff }}$, the use of a handover algorithms causes in increase from $1.98 \mathrm{~dB}$ to $2.83 \mathrm{~dB}$ when $\mathrm{E}_{\text {diff }}<3$, and an increase from $1.63 \mathrm{~dB}$ to $2.14 \mathrm{~dB}$ when $3<\mathrm{E}_{\text {diff }}<6$.

However, most of the samples where an increase in SFNG is noticed are low quality samples (MFER more than 10\%), which might be less likely to appear in actual deployed networks. When applying the same criterion as in [1] for link budget calculations (only retaining doubtful and good quality samples where $E_{\text {diff }}<9 \mathrm{~dB}$ ), only a slight SFNG increase is noticed. Table III shows that in the ideal scenario, a median SFN gain of $1.08 \mathrm{~dB}$ is obtained, compared to a median SFN gain of $1.13 \mathrm{~dB}$ in the handover scenario. When only retaining good and doubtful quality segments where $\mathrm{E}_{\text {diff }}<6 \mathrm{~dB}$, the influence of the handover mechanism is higher: $1.70 \mathrm{~dB}$ vs. $1.44 \mathrm{~dB}$. It should be noted that of course, the use of handover

\begin{tabular}{|r||l|l|}
\hline \multicolumn{2}{|c|}{$\begin{array}{r}\text { SFNG } \\
\text { (\# samples [-]) }\end{array}$} & \multicolumn{2}{|c|}{ Scenario } \\
\cline { 2 - 3 } Perfect & With handover & Ideal \\
\hline \hline Good & $0.91(46)$ & $-2.87(124)$ \\
\hline Doubtful & $2.42(13)$ & $2.20(12)$ \\
\hline Low & $3.08(204)$ & $2.24(203)$ \\
\hline \hline $\begin{array}{r}\text { SFNG } \\
\text { (\# samples }[-])\end{array}$ & \multicolumn{2}{|c|}{ Scenario } \\
\cline { 2 - 3 } With handover & Ideal \\
\hline \hline $\mathrm{E}_{\text {diff }}<\mathbf{3}$ & $2.83(167)$ & $1.98(167)$ \\
\hline $\mathbf{3}<\mathrm{E}_{\text {diff }}<\mathbf{6}$ & $2.14(58)$ & $1.63(58)$ \\
\hline $\mathbf{6}<\mathrm{E}_{\text {diff }}<\mathbf{9}$ & $1.35(47)$ & $1.35(47)$ \\
\hline $\mathbf{9}<\mathrm{E}_{\text {diff }}$ & $-3.04(115)$ & $-3.04(115)$ \\
\hline
\end{tabular}

TABLE II

COMPARISON OF MEDIAN SFNG SFNG med $_{\text {(AND NUMBER OF }}$ RECORDED SAMPLES) FOR THE IDEAL SCENARIO AND THE HANDOVER SCENARIO FOR FOUR DIFFERENT QUALITY CATEGORIES [1] AND FOR FOUR DIFFERENT OVERLAPPING CATEGORIES [1].

\begin{tabular}{|r|r|r|r|}
\hline \multicolumn{5}{|c|}{ Good and doubtful segments, $\mathrm{E}_{\text {diff }}<9$} \\
\hline Scenario & SFNG $_{\text {med }}[\mathbf{d B}]$ & \# samples [-] & $\sigma[\mathbf{d B}]$ \\
\hline Ideal & 1.08 & 44 & 3.26 \\
\hline Handover & 1.13 & 43 & 3.33 \\
\hline \multicolumn{4}{|c|}{ Good and doubtful segments, $\mathrm{E}_{\text {diff }}<6$} \\
\hline Scenario & SFNG med $_{\text {med }}[\mathbf{d B}]$ & \# samples [-] & $\sigma[\mathbf{d B}]$ \\
\hline Ideal & 1.44 & 23 & 3.47 \\
\hline Handover & 1.70 & 22 & 3.60 \\
\hline
\end{tabular}

TABLE III

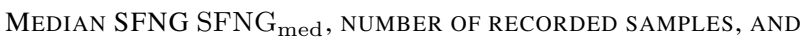
STANDARD DEVIATION OF SEGMENTS RETAINED FOR LINK BUDGET CALCULATION FOR TWO DIFFERENT CASES.

mechanisms do not cause an actual increase of the SFNG, they just incorporate another (more realistic) definition of SFN gain. This study indicates that in realistic scenarios (with handover mechanisms), the actual SFNG values might be slightly higher than expected based on [1], but this is mainly the case in areas that are not well covered and where in the ideal scenario, a lot of handovers occur.

At first sight, it might seem like a good approach to decrease the window size from $100 \mathrm{~m}$ to e.g., $50 \mathrm{~m}$, to have more flexibility in the choice of handover scenario. However, for smaller window sizes, it is far more difficult to obtain a reliable window synchronization between the different scenarios [1]. Also, it is important to maintain a sufficient averaging of small-scale fading within one segment. This should be kept in mind when applying other handover scenarios.

\section{B. Influence of Transmission Delay Difference on SFNG}

In this section, the the link between the SFN gain and the transmission delay difference experienced at the receiver will be investigated. No handover mechanisms have been applied for this analysis. Figs. 3, 4, 5 show the SFN gain as a function of $E_{\text {diff }}$, as a function of the time arrival differences $\triangle_{1}$ between the direct rays from the two most dominant transmitters, and as a function of the time arrival 


\begin{tabular}{|r|r||r|r||r|r|}
\hline $\begin{array}{r}E_{\text {diff }} \\
{[\mathrm{dB}]}\end{array}$ & $\begin{array}{r}\mathrm{SFNG}_{\mathrm{md}}[\mathrm{dB}] \\
\text { (\# samples [-]) }\end{array}$ & $\begin{array}{r}\triangle_{1} \\
{[\%]}\end{array}$ & $\begin{array}{r}\mathrm{SFNG}_{\mathrm{md}}[\mathrm{dB}] \\
\text { (\# samples [-]) }\end{array}$ & $\begin{array}{r}\triangle_{2} \\
{[\%]}\end{array}$ & $\begin{array}{r}\mathrm{SFNG}_{\mathrm{md}}[\mathrm{dB}] \\
\text { (\# samples [-]) }\end{array}$ \\
\hline \hline $0-5$ & $2.01(207)$ & $0-5$ & $3.57(43)$ & $0-5$ & $0.70(10)$ \\
\hline $5-10$ & $1.13(77)$ & $5-10$ & $0.33(134)$ & $5-10$ & $1.41(101)$ \\
\hline $10-15$ & $-0.68(48)$ & $10-15$ & $1.01(163)$ & $10-15$ & $1.57(136)$ \\
\hline $15-20$ & $-3.49(28)$ & $15-20$ & $-1.69(33)$ & $15-20$ & $-1.03(82)$ \\
\hline $20-25$ & $-4.81(19)$ & $20-25$ & $-1.34(14)$ & $20-25$ & $0.22(58)$ \\
\hline $25-30$ & $-3.24(8)$ & & & \multicolumn{3}{|c|}{} \\
\hline
\end{tabular}

TABLE IV

MEDIAN SFNG SFNG md $_{\text {FOR DIFFERENT INTERVAL OF THE VARIABLES }}$ $\mathrm{E}_{\text {diff }}, \triangle_{1}$, AND $\triangle_{2}$, AND NUMBER OF SAMPLES RECORDED WITHIN EACH INTERVAL.

differences $\triangle_{2}$ of the direct rays from the closest and the furthest transmitter. These time differences are expressed as percentages of the guard interval length corresponding with the aforementioned transmit signal settings.

The figures show that the SFNG decreases with $\mathrm{E}_{\text {diff }}$, and also (though to a lesser extent) with $\triangle_{1}$ and $\triangle_{2}$, as can be expected.

Table IV shows the median SFN gain $\mathrm{SFNG}_{\mathrm{md}}$ as a function of the $E_{\text {diff }}$ interval and as a function of the signal arrival difference interval. This table and Figs. 3, 4, 5 show that the decreasing trend is most apparent for increasing values of $\mathrm{E}_{\text {diff. }}$.

Although all three variables $\left(E_{\text {diff }}, \triangle_{1}\right.$, and $\left.\triangle_{2}\right)$ have an inverse relationship with the SFNG, we have calculated the correlation coefficient between each of the three variables and the SFNG, in order to numerically assess their relationship. It was shown that $E_{\text {diff }}$ is a better predictor than the other two quantities (correlation coefficient of -0.48 vs. correlation coefficient of both -0.24). Therefore, when deploying an SFN, $\mathrm{E}_{\text {diff }}$ is a more interesting variable to characterize $\mathrm{SFN}$ gain than $\triangle_{1}$ or $\triangle_{2}$.

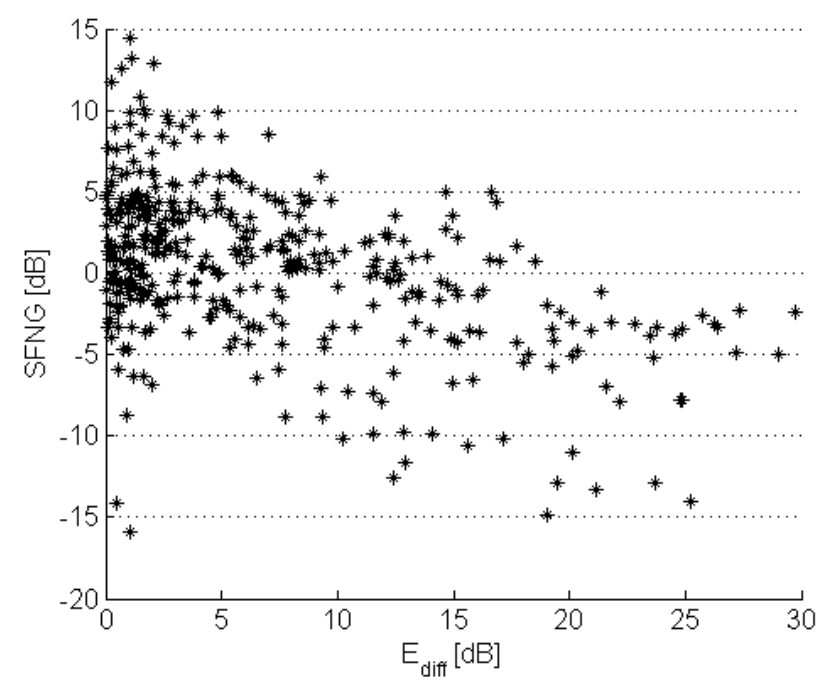

Fig. 3. SFNG $[\mathrm{dB}]$ as a function of overlapping degree $\mathrm{E}_{\mathrm{diff}}[\mathrm{dB}]$.

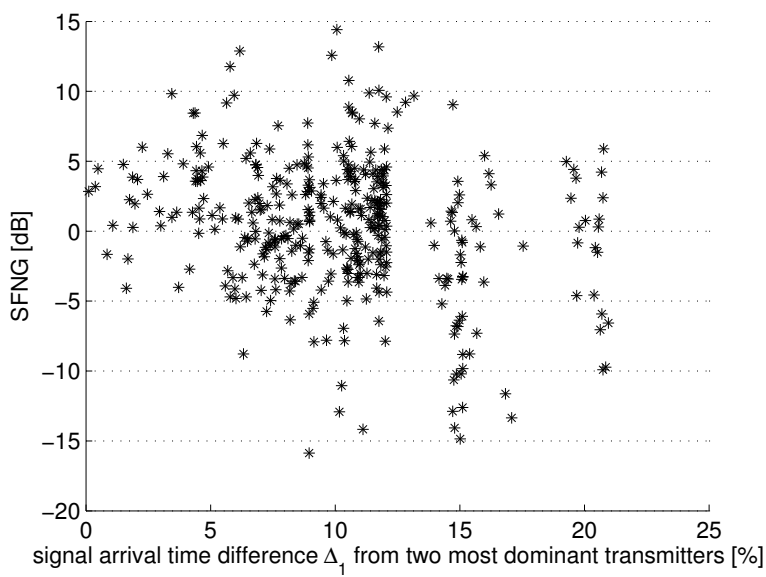

Fig. 4. SFNG $[\mathrm{dB}]$ as a function of the time arrival difference $\triangle_{1}$ between the direct rays from the two most dominant transmitters, expressed as a percentage of the guard interval [\%].

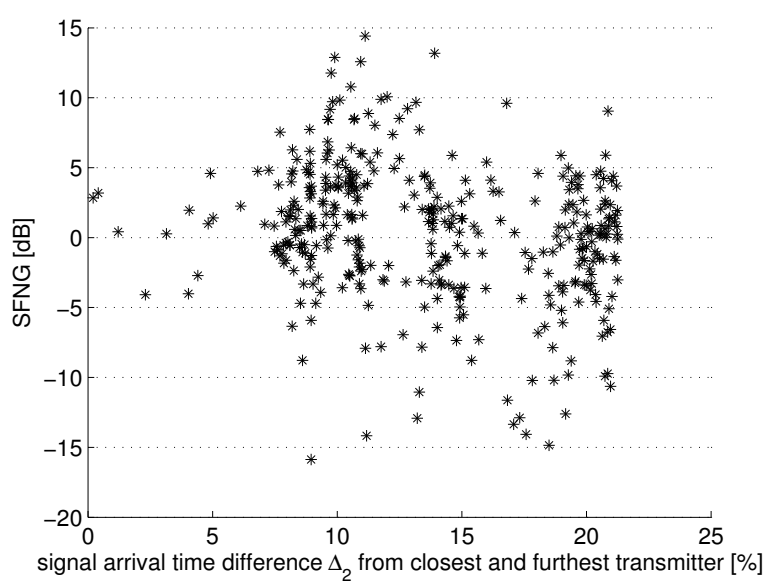

Fig. 5. SFNG $[\mathrm{dB}]$ as a function of the time arrival difference $\triangle_{2}$ between the direct rays from the closest and the furthest transmitter, expressed as a percentage of the guard interval [\%].

\section{Conclusion}

In this paper we have proposed extensions and new approaches for SFN gain calculations. A more realistic definition for the SFN gain was proposed, incorporating a handover mechanism at the receiver. The new definition yielded a small increase in SFN gain compared to the 'ideal' situation, where the receiver automatically switches to the best serving transmitter. The study indicates that in realistic scenarios (with handover mechanisms), the actual SFNG values might be slightly higher than expected based on [1], but this is mainly the case in areas that are not well covered and where in an ideal scenario, a lot of handovers would occur. Other research indicated that the 'overlapping degree' $E_{\text {diff }}$ is a better predictor for the SFN gain than the transmission delay difference between the signals from the different transmitters: respective correlation coefficients of -0.48 and -0.24 have been 
obtained.

\section{ACKNOWLEDGMENT}

This work was supported by the IBBT - MADUF (Maximize DVB Usage in Flanders) project, co-funded by the IBBT (Interdisciplinary institute for BroadBand Technology), a research institute founded by the Flemish Government in 2004, and the involved companies and institutions. W. Joseph is a Post-Doctoral Fellow of the FWO-V (Research Foundation - Flanders).

\section{REFERENCES}

[1] D. Plets, W. Joseph, P. Angueira, J. Arenas, L. Verloock, and L. Martens, "On the Methodology for Calculating SFN Gain in Digital Broadcast Systems," Broadcasting, IEEE Transactions on, vol. 56, no. 3, pp. 331 -339 , sept. 2010

[2] ETSI, TR 102401 v1.1.1, "Digital Video Broadcasting (DVB); Transmission to Handheld Terminals (DVB-H); Validation Task Force Report," May 2005.

[3] X. Yang, J. Vare, and T. Owens, "A survey of handover algorithms in dvb-h," Communications Surveys Tutorials, IEEE, vol. 8, no. 4, pp. 16 -29 , quarter 2006. 